\title{
O AuXILIAR Aspectual TyKa DO KARITIANA
}

The aspectual auxiliary tyka in Karitiana

\author{
Andrea Marques de Carvalho*
}

\section{INTRODUÇÃO}

Este artigo traz uma análise semântica da palavra tyka do Karitiana, língua amazônica da família Arikém (tronco Tupi). Tendo o aspecto verbal dessa língua como foco geral e o auxiliar tyka como foco particular, nossas principais questões dizem respeito à interpretação desse verbo, à forma como ele opera nas sentenças e ao que seu comportamento indica sobre $o$ aspecto verbal em geral na língua.

Defendemos que tyka é um auxiliar aspectual compatível apenas com sentenças de TT $\geq \mathrm{TU}$ (tempo do tópico, de referência, posterior ou simultâneo ao tempo da fala) e que representa a relação aspectual de TT $\underline{C}$ TSit (tempo do tópico contido no tempo do evento), correspondente à representação dada para o imperfectivo (cf. KLEIN, 1994). Defendemos também que, além de marcar a imperfectividade - tanto episódica (de eventos singulares) quanto habitual ou iterativa (de eventos plurais) - em sentenças de tempo de tópico presente ou futuro, tyka, mais especificamente seu morfema $-k a$, opera sobre um dos nominais, selecionando apenas as entidades atômicas de sua denotação. De acordo com Landin (1984), ele também pode expressar a posição do corpo da referência desse mesmo nominal ao substituir o morfema -ka por-so (em pé), -syp (deitado) ou -ja (sentado).

Organizamos este artigo da seguinte maneira: Na seção 2, apresentamos o pano de fundo teórico; na seção 3, citamos algumas considerações relevantes sobre a gramática do Karitiana; em 4, apresentamos a análise feita para cada uma das hipóteses levantadas; em 5, sugerimos uma forma lógica que descreva a semântica de uma sentença construída

Universidade de São Paulo 
com o tyka e, em 6, finalmente, concluímos com um resumo dos resultados obtidos.

\section{PANO DE Fundo TEÓRICO}

O objetivo desta seção é explicitar toda a base teórica utilizada em nossa análise. Primeiramente, apresentamos o teste de auxiliaridade proposto por Haegemann (1995); em seguida, apresentamos algumas abordagens formais do imperfectivo, a organização das classificações aspectuais sugerida por Castilho e Moraes de Castilho (1994), o tratamento do aspecto oferecido pela semântica de eventos e as adaptações de Jóhannsdóttir (2006); por fim, apresentamos a abordagem dos operadores selecionadores de entidades atômicas nas denotações dos nomes comuns (doravante NCs) sugerida por Paraguassu-Martins e Müller (2008).

Considerando a hipótese de que tyka é um auxiliar, utilizamos um dos principais testes de auxiliaridade encontrados na literatura. Haegemann (1995) afirma que, diferentemente de um verbo pleno, um auxiliar jamais atribui papel temático. Uma sentença com um único verbo e outra com um auxiliar incluído devem conter o mesmo número de NPs, o mesmo número de papéis temáticos atribuídos. Vale ressaltar que, sendo tal teste baseado na teoria gerativista, considera-se que existem papéis temáticos e que cada NP de uma sentença pode e precisa receber apenas um desses papéis.

Considerando o valor imperfectivo que atribuímos ao tyka, recorremos à perspectiva da Semântica Formal quanto à questão do aspecto. Na literatura formal, há um consenso de que o aspecto reflete a relação entre o momento de um evento e um momento de referência. Com base em ideias de Reichenbach (1947), Klein (1994) reconhece que os morfemas de tempo e aspecto relacionam cronologicamente três momentos: TU (momento da fala), TSit (momento do evento, da situação) e TT (momento de referência, de tópico). A relação entre o TT e o TSit definiriam o aspecto em uma sentença:

\section{PERfECTIVO}

TT AT TSit (com o TT parcialmente incluído no TSit)

Ex.: Eu dormi.

No exemplo acima, o TT não é explícito na sentença, ele seria dado pelo contexto, como em resposta à pergunta "O que você fez entre $5 \mathrm{~h}$ e 5 h30 ontem?". Esse TT poderia estar, por exemplo, parcialmente anterior ao TSit (tempo do sono de quem produz a sentença), como no caso de o falante ter acordado antes do término do período estabelecido; poderia estar parcialmente posterior a esse TSit, como no caso de o falante ter caído no 
sono às cinco e quinze; ou poderia, ainda, conter todo o TSit, como no caso de o falante ter dormido entre as cinco e dez e as cinco e vinte.

\section{IMPERFECTIVO}

TT INCL TSit (com o TT totalmente incluído no TSit)

Ex.: Eu estava trabalhando ontem às duas horas.

No exemplo acima, o TT (ontem às duas horas) está dentro de um período maior, o TSit (tempo em que o falante trabalhou).

\section{PRospectivo}

TT BEFORE TSit (com o TT anterior ao TSit)

Ex.: Ela ainda ia almoçar.

No exemplo acima, o TT (dado pelo contexto) é anterior ao TSit (o momento do almoço da pessoa sobre quem se fala).

\section{PERfEITO}

TT AFTER TSit (com o TT posterior ao TSit)

Ex.: Às nove horas eu já tinha acordado.

No exemplo acima, o TT (nove horas) é posterior ao TSit (momento em que o falante acorda).

Voltando nossa atenção para o imperfectivo, ressaltamos que uma das características do imperfectivo apontada por Klein (1994) é a sua incompatibilidade com sentenças de VPs estativos de conteúdo lexical nãotemporário. Tais conteúdos são aqueles que se mantêm inalterados em qualquer TT, o que impossibilita uma diferenciação aspectual. O TSit de sentenças com verbos que apresentam esse tipo de conteúdo sempre inclui qualquer TT. Observe que a sentença em (1) é estativa, assim como aquela em (2). No entanto, (1) é perfeitamente gramatical, enquanto (2) não o é.

$$
\begin{aligned}
& \text { (1) João está sendo sincero. } \\
& \text { (2) João está sendo brasileiro. }
\end{aligned}
$$

É importante destacar que, além do valor qualitativo do aspecto verbal (perfectivo vs. imperfectivo), há também uma perspectiva quantitativa. Essa perspectiva permite leituras episódicas (denotando um evento), habituais (denotando um número indeterminado e maior que um de eventos que se repetem) ou iterativas (denotando um número determinado e maior que um de eventos que se repetem) (cf. CASTILHO; MORAES DE CASTILHO, 1994). 
Considerando nossa hipótese de que o tyka não traz apenas informação aspectual, operando também sobre a denotação de um nominal, a fórmula TT $\underline{\mathrm{C}}$ TSit, representação do imperfectivo, segundo Klein (1994), representaria apenas parte da semântica desse auxiliar. A semântica de eventos oferece uma descrição formal para sentenças inteiras capaz de absorver todos os detalhes semânticos do tyka.

Partindo de Davidson (1967), Parsons (1990) apresenta formas lógicas para sentenças caracterizadas sintaticamente. Utilizando o operador Hold em oposição ao Cul para representar a perduração em oposição à culminação de um evento e separando os argumentos da denotação do verbo, a sentença (3) teria em (4) sua representação formal:

(3) Ágata estava atravessando a rua.

(4) $\quad(? \mathrm{t})(\mathrm{t}<\mathrm{agora} \&(? \mathrm{E})$ [atravessar $(\mathrm{E}) \&$ sujeito (E, Ágata) $\&$ objeto (E, a rua) \& Hold (E, t)

(Existe um tempo anterior ao agora e existe um evento de atravessar, cujo sujeito é Ágata e cujo objeto é a rua. Este evento perdura no tempo mencionado)

É interessante notar que o raciocínio de Parsons (1990) é compatível

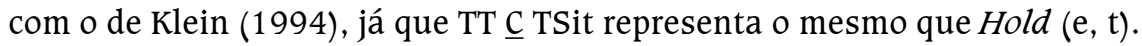

Ao representar a imperfectividade de uma sentença em uma forma lógica por meio do operador Hold, mesmo operador utilizado para representar sentenças estativas, Parsons (1989) interpreta o imperfectivo como estativizador de eventos. Considerando tais constatações, Jóhannsdóttir (2006) trata os verbos progressivos como estados para representar as sentenças progressivas com verbos posturais do islandês. A sentença (5) teria em (6) sua representação formal.

$$
\begin{aligned}
& \text { Jón situr og les. } \\
& \text { Jón senta e lê. } \\
& \text { Contexto: “Jón está (sentado e) lendo." } \\
& \text { ?s ( Sentar (s) \& Ler (s) \& Holder (jón) (s)) }
\end{aligned}
$$

Jóhannsdóttir (2006) utiliza a nomenclatura de Kratzer (1994). Holder seria o argumento externo de um estado em oposição a Agent, argumento externo de um evento.

Por fim, levando em conta a hipótese de que tyka é um operador que singulariza um dos nominais, voltemos nossas atenções ao tratamento de Paraguassu-Martins e Müller (2008) para o operador singular. As autoras 
consideram que a denotação dos NCs no português brasileiro é neutra em relação a número. Se alguém diz que João comprou maçã, ele pode ter comprado tanto uma quanto duas ou mais. Considerando uma denotação neutra para os NCs, Paraguassu-Martins e Müller (2008) defendem que o singular e o plural são operações sobre tal neutralidade (cf. MÜLLER, 2001). O operador singular selecionaria as entidades atômicas e o plural, o conjunto das pluralidades.

\section{A GRAMÁTICA DO KARITIANA}

O objetivo desta seção é pontuar algumas questões gramaticais já conhecidas do Karitiana que se mostrarão relevantes para a compreensão deste trabalho. Inicialmente, apresentamos o núcleo dessa língua, formado pelo verbo principal e pelo verbo auxiliar aspectual; em seguida, apresentamos um resumo da morfologia do aspecto e, por fim, introduzimos a questão da pluracionalidade na língua.

De acordo com Storto (1999), o Karitiana é uma língua de verbo em posição final que apresenta movimento verbal obrigatório nas orações principais para que tempo e concordância sejam atribuídos. Storto (1999) acrescenta que o verbo e o auxiliar aspectual formam um núcleo complexo que ocupa a segunda posição das orações principais. Gerado em posição final, o auxiliar aspectual também é alçado e se junta à direita do verbo, assumindo a segunda posição. Em uma oração principal sem núcleo aspectual superficial, além de trazer marcação de tempo e de concordância absolutiva, o verbo também traz marcação de modo (cf. STORTO, 2002). Em uma oração principal com um auxiliar aspectual, o verbo mantém a concordância e o modo, mas é o auxiliar aspectual que traz a marca de tempo.

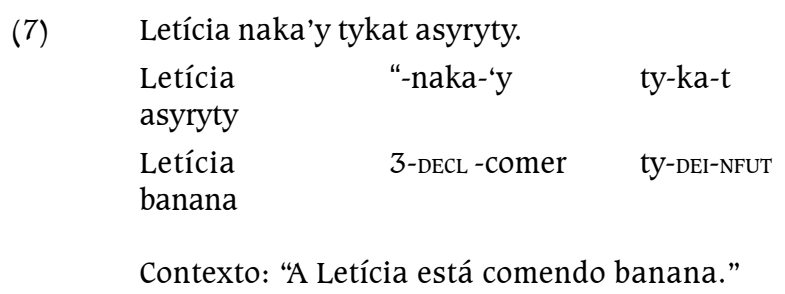

Storto (2002) defende que os marcadores aspectuais abertos do Karitiana podem ser divididos em dois grupos, os clíticos e os auxiliares. No primeiro grupo estão o iterativo, o inceptivo e o dubitativo. No segundo estão o referencial e o imperfectivo. Este, marcado por ty+dêitico, é o aspecto verbal do Karitiana mais citado na literatura. Classificado como auxiliar 
imperfectivo por Storto (2002), é tratado como sufixo progressivo por Landin (1984) e por Everett (2006). De qualquer forma, há um consenso de que tal marcador pode trazer em sua semântica a indicação da posição do corpo da referência de um nominal. Tyka indicaria "movimento"; tyso, "em pé"; tysyp, "deitado (também plural)" e tyja, "sentando". Segundo Everett (2006), tais marcadores ocorrem apenas em sentenças no presente.

Outra hipótese bastante relevante para este estudo é defendida por Müller e Sanchez-Mendes (2008). As autoras afirmam que raízes nominais e verbais em Karitiana têm denotações cumulativas, e que os afixos pluracionais - a reduplicação verbal - atuam como uma operação plural sobre as denotações dos verbos, excluindo os eventos singulares.

\section{ANÁLISE}

Pretendemos, neste momento do artigo, submeter a palavra tyka do Karitiana a uma análise que parte do suporte teórico e das descrições desta língua apresentados acima. Em 4.1., analisamos a auxiliaridade do tyka; em 4.2., investigamos seu caráter imperfectivo; em 4.3., avaliamos sua gramaticalidade em sentenças de eventos singulares e plurais; em 4.4., analisamos sua possível restrição com determinados tempos verbais; em 4.5., conferimos seu papel como auxiliar postural; e, por fim, em 4.6., analisamos a hipótese de que tyka seleciona apenas as entidades atômicas da denotação de um nome.

Veremos a seguir que tyka é um verbo auxiliar bimorfêmico incompatível com sentenças no passado. Veremos que seu morfema tyrepresenta a relação de TT $\underline{\mathrm{C}}$ TSit, marcando a imperfectividade de eventos singulares ou plurais, e que seu morfema $-k a$ singulariza um nominal, atribuindo a este um certo movimento corporal.

\subsection{TYKA COMO AUXILIAR}

O objetivo desta seção é mostrar que a palavra tyka do Karitiana não é um verbo pleno, mas sim um verbo auxiliar. Na literatura, notamos que duas classificações são atribuídas ao tyka. Storto (2002) o trata como auxiliar, enquanto Everett (2006) o trata como sufixo verbal. A segunda hipótese já é prontamente descartada pelo fato de que a palavra tyka tem acento próprio. Nossa questão, portanto, é se ele é de fato um auxiliar ou se poderia ser, na verdade, um verbo pleno.

Defendemos a hipótese de que tyka é, como afirma Storto (2002), um auxiliar. Nossa hipótese encontra suporte em um dos testes de auxiliaridade propostos por Haegemann (1995). Como verbos auxiliares, 
diferentemente dos verbos plenos, não atribuem papel temático, os sintagmas nominais de uma sentença em Karitiana com um verbo pleno e o tyka devem ser apenas aqueles necessários para que as exigências temáticas do verbo principal sejam satisfeitas.

O contraste entre as sentenças abaixo é uma evidência de que nossa hipótese está correta. Comparando as sentenças (8a) e (8b), observamos que o número de NPs é exatamente o mesmo.

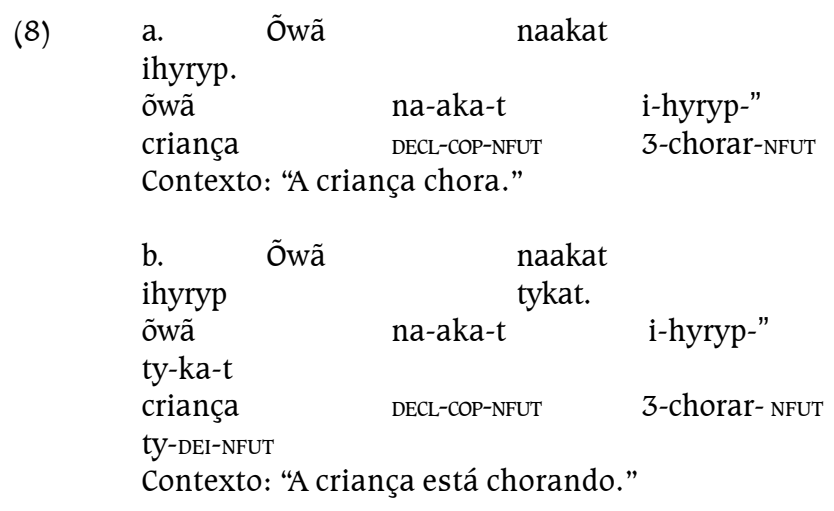

Como (8b) não traz outro NP além daquele presente em (8a), podemos concluir que tyka não atribui papéis temáticos e, portanto, é um auxiliar. Vale ressaltar que o verbo principal e o tyka formam um grupo indissociável - nenhuma palavra pode separá-los - e que este, mas não o primeiro, traz um sufixo de tempo. Isso reforça, ainda mais, a hipótese de que tyka é de fato um auxiliar.

\subsection{TyKa Sob Uma Perspectiva Qualitativa Apectual: Um Auxiliar MARCADOR DA IMPERFECTIVIDADE}

O objetivo desta seção é identificar a natureza aspectual do tyka. Há outra divergência em torno dele, já que Storto (2002) o classifica como auxiliar imperfectivo, enquanto Everett (2006), seguindo Comrie (1985), o trata como marcador do progressivo, subcategoria do imperfectivo. Nesta análise adotamos a teoria de Klein (1994) e consideramos que aspecto verbal diz respeito à relação entre TT (tempo de tópico) e TSit (tempo do evento). Nossa questão, portanto, é identificar qual relação entre TT e TSit o auxiliar tyka seleciona. Nossa hipótese é que ele seleciona a relação de TT INCL TSit, (tempo de tópico incluído no tempo do evento), indicando a imperfectividade da sentença. 
De acordo com Klein (1994), o imperfectivo é incompatível com VPs que tragam verbos estativos de conteúdo lexical não-temporário. Esse teste aplicado ao Karitiana parece corroborar a nossa hipótese. Observe o contraste entre as sentenças abaixo.

$\begin{array}{llll}\text { Õwã } & \text { naakat } & \text { iokõrong } & \text { tykat. } \\ \text { Õwã } & \text { na-aka-t } & \text { i-okõrong } & \text { ty-ka-t. } \\ \text { Criança } & \text { DECL-coP-NFUT } & \text { 3-forte } & \text { ty-DEI-NFUT }\end{array}$

Contexto: "A criança está sendo forte (emocionalmente)."

$\begin{array}{llrl}* \text { Pedro } & \text { naakat } & \text { americano } & \text { tykat. } \\ \text { Pedro } & \text { na-aka-t } & \text { americano } & \text { ty-ka-t. } \\ \text { Pedro } & \text { DECL-COP-NFUT } & \text { americano } & \text { ty-DEI-NFUT } \\ \text { Contexto: "O Pedro é americano." } & \end{array}$

Observando os dados acima, podemos concluir que o auxiliar tyka é imperfectivo, pois ele coloca o TT dentro do TSit e, se testado com sentenças de verbos de conteúdo lexical não-temporário, ele as torna agramaticais, já que nesse tipo de ambiente não há contraste de TTs possível para que haja alguma diferenciação aspectual.

\subsection{TyKa SOB UmA PERSPECTIVA QUANTITATIVA: Um AUXILIAR IMPERFECTIVO PARA EVENTOS EPISÓdICOS, HABITUAIS E ITERATIVOS}

Considerando que o imperfectivo permite diferentes tipos de leituras, o objetivo desta seção é identificar os tipos de leituras possíveis de uma sentença com tyka sob a perspectiva aspectual quantitativa. Em relação a esta questão, há, mais uma vez, divergência na literatura. Storto (2002) afirma que o tyka não ocorre apenas em eventos episódicos, mas também em sentenças habituais e estativas. Por outro lado, Everett (2006) defende que o auxiliar em estudo jamais ocorre em sentenças que indiquem hábito. Nossa hipótese, em concordância com Storto (2002), é de que tanto as leituras de eventos singulares quanto as de eventos plurais são permitidas pelo auxiliar tyka.

A interpretação de um evento descrito em uma sentença do Karitiana com tyka como singular ou plural pode ser direcionada por meio de recursos como a reduplicação verbal, advérbios ou informação contextual. Uma sentença sem nenhum desses recursos explícitos, entretanto, pode apontar tanto para um evento singular quanto para um evento plural. Observe o exemplo em (11). 


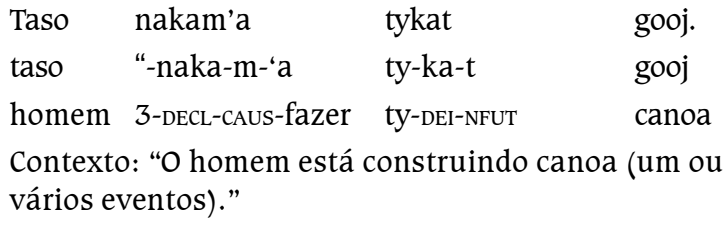

A sentença em (11) permite, além da leitura episódica, a leitura plural. Ela pode ser aplicada, por exemplo, em um contexto em que um homem que costumava criar animais decide mudar de atividade e passa a construir canoas.

Se nossa hipótese estiver correta, podemos prever que o uso do tyka seria gramatical não apenas em sentenças com um único evento, mas também em sentenças com advérbios que selecionem eventos plurais, como "recentemente". As sentenças abaixo comprovam nossa hipótese:

$$
\begin{aligned}
& \text { a. Dibm duas horas taso nakam'a } \\
& \text { tykat gooj. } \\
& \text { dibm duas horas taso "-nakam'a } \\
& \text { ty-ka-t gooj } \\
& \text { amanhã duas horas homem 3-DECL-CAus-fazer } \\
& \text { ty-DEI-NFUT canoa } \\
& \text { Contexto: "Amanhã às duas horas o homem vai estar } \\
& \text { construindo canoa." } \\
& \text { b. Ka'it pymbyrat taso nakam'a } \\
& \text { tykat gooj. } \\
& \text { Ka'it pymbyrat taso "-naka-m-'a } \\
& \text { ty-ka-t gooj. } \\
& \text { Recentemente homem 3-DECL-CAus-fazer } \\
& \text { ty-DEI-NFUT canoa }
\end{aligned}
$$

Com base na gramaticalidade dos dados acima, podemos concluir que as sentenças do Karitiana construídas com o auxiliar tyka podem ser interpretadas não apenas como descrições de eventos singulares (episódicos), mas também como descrições de eventos plurais (habituais ou iterativos).

\subsection{A Restrição do Uso do AuXiliar TyKa Para SentençAS de Tempo DE Tópico Posterior ou IGUAL ao TEMPo DA FALA}

O objetivo desta seção é conferir em quais relações entre TT e TU o uso do tyka é gramatical. Everett (2006) defende que o uso deste auxiliar, 
tratado por ele como sufixo, é possível apenas em sentenças que descrevem situações no presente, quando o TT é simultâneo ao TU. Nossa questão é se tal restrição de fato procede e, se a resposta for positiva, como seria marcada a imperfectividade nos demais casos. Nossas constatações mostram que $o$ tyka é incompatível apenas com sentenças que descrevem situações no passado - em que o TT é anterior ao TU. Sentenças para situações futuras em que o TT é posterior ao TU - seriam perfeitamente possíveis. Tyka apontaria para uma distinção temporal entre presente e passado não marcada pelos morfemas de tempo do Karitiana.

Se a hipótese de Everett (2006) estivesse correta, sentenças formuladas com o tyka, se marcadas com advérbios que ancoram o TT anterior ou posteriormente ao TU, deveriam ser agramaticais. No entanto, não é isso que observamos nos dados abaixo. Não é apenas a sentença em (13a), cujo momento de referência é simultâneo ao da fala, que é aceita como gramatical. Observamos a mesma gramaticalidade na sentença em (13c), cuja descrição é de um evento com referência explicitamente ancorada em um momento posterior ao da fala por meio da inclusão do advérbio "dibm duas horas" - amanhã às duas horas.

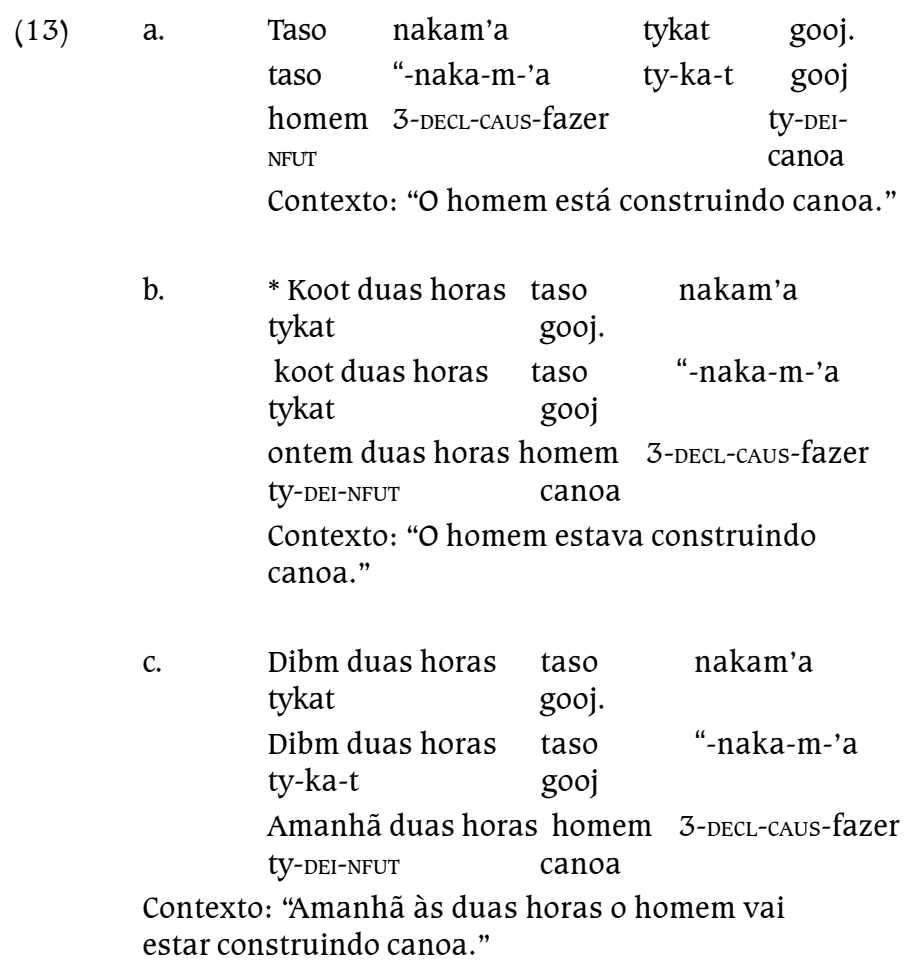


Os dados indicam que o uso do tyka é incompatível com sentenças de TT anterior ao TU. Nesses casos, outro auxiliar é utilizado para a marcação da imperfectividade do evento:

$$
\begin{array}{lc}
\text { Taso } & \text { nakam'a } \\
\text { andyk } & \text { gooj. } \\
\text { taso } & \text { "-naka-m-'a } \\
\text { andyk } & \text { gooj } \\
\text { homem } & \text { 3-DECL-CAUs-fazer } \\
\text { gooj } & \\
\text { Contexto: "O homem estava construindo canoa." }
\end{array}
$$

Com base nos dados acima, podemos concluir que a restrição do uso do tyka não é para sentenças de TT $=$ TU, mas sim para sentenças de TT $\geq$ TU. É curioso notar que, apesar de o Karitiana não apresentar uma distinção entre passado e presente por meio da morfologia verbal, tal distinção é feita por meio do uso de auxiliares aspectuais.

\subsection{TYKA COMO AUXILIAR POSTURAL}

O objetivo desta seção é apresentar e confirmar a interpretação postural das possíveis substituições morfêmicas do auxiliar tyka. Landin (1984) identifica uma série de morfemas que podem substituir - $k a$ para indicar a posição corporal da referência de um nominal de uma sentença imperfectiva. Não parece haver discordância em relação a esse tema na literatura. Portanto, nossa questão é simplesmente confirmar ou não a descrição feita por Landin (1984) dos morfemas substitutos e das posturas que cada um deles representa. Defendemos que Landin (1994) está correto em suas afirmações.

Os morfemas que podem substituir o-ka do tyka para expressar a

\begin{tabular}{|c|c|c|c|}
\hline \multirow[t]{3}{*}{$(15)$} & $\begin{array}{l}\text { Maria } \\
\text { kinda'o. }\end{array}$ & naka’y & tykat / tysot / tyjat / tysyp \\
\hline & $\begin{array}{l}\text { Maria } \\
\text { ty-syp-" }\end{array}$ & $\begin{array}{l}\text { “-naka-’y } \\
\text { kinda'o }\end{array}$ & ty-ka-t / ty-so-t / ty-ja-t / \\
\hline & $\begin{array}{l}\text { Maria } \\
\text { fruta }\end{array}$ & 3-DECL-Comer-NFUT & ty-DEI-NFUT \\
\hline
\end{tabular}
posição corporal da referência de um nominal são: -so, -syp e -ja. O morfema -ka é o uso neutro do auxilar, enquanto -so indica "em pé", -syp, "deitado" e -ja, "sentado" (cf. LANDIN, 1984).

Durante nosso trabalho de campo, coletamos dados com as diferentes possibilidades de ty+dêitico. Uma sentença imperfectiva pode apresentar as seguintes variações: 
Contexto: "Maria está comendo fruta em movimento/em pé/sentada/deitada."

É importante ressaltar que tysyp pode indicar que a referência de um nominal "está sentada" em alguns contextos ou que é plural em outros, de acordo com Landin (1984), Storto (2002) e Everett (2006). Um estudo mais detalhado a respeito ainda precisa ser realizado.

\section{6. - KA COMO UM OPERAdor QUe Seleciona Apenas Entidades ATÔMICAS DA DENOTAÇÃo DOS NOMES COMUNS}

O objetivo desta seção é mostrar como o morfema - $k a$ do auxiliar tyka atua sobre as denotações dos nomes. Ao descrever as possíveis substituições morfêmicas do auxiliar tyka, Landin (1984) identifica uma das opções, o tysyp, como indicadora da pluralidade do nominal absolutivo. Em nossos trabalhos de campo, notamos que o uso de tyka é inaceitável em sentenças transitivas em que ambos os nominais são semanticamente plurais, como no caso da sentença abaixo, assim como em sentenças intransitivas em que seu único nominal é plural.

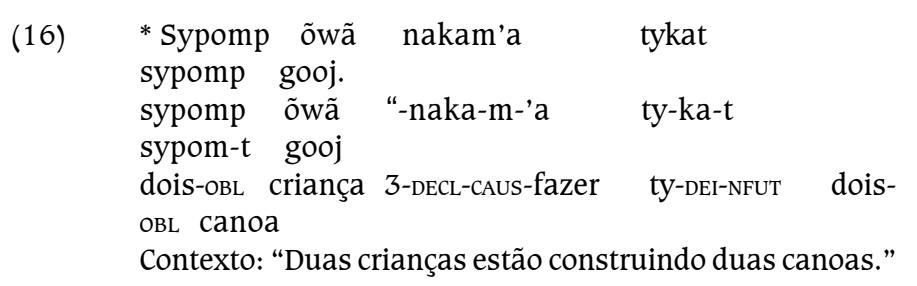

Müller et al. (2006) defendem que nomes em Karitiana são cumulativos. Os sintagmas nominais não seriam marcados para número e seriam livres de material funcional. Uma sentença com argumentos nus seria verdadeira para qualquer número de entidades do tipo apropriado. A sentença abaixo, por exemplo, possibilita uma série de leituras. Poderia indicar que um homem comeu uma cobra, ou que mais de um homem comeu uma cobra, ou que um homem comeu mais de uma cobra, ou até mesmo que mais de um homem comeu mais de uma cobra.

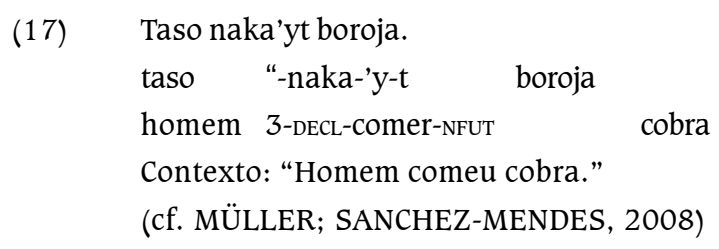


Nossa questão é como o morfema - $k a$ do auxiliar imperfectivo tyka pode operar sobre a denotação desses nomes. Defendemos a hipótese de que ele opera sobre um nominal, selecionando apenas entidades atômicas de sua denotação.

Com base na afirmação de Paraguassu-Martins e Müller (2008) de que os NCs do português têm uma denotação neutra em relação ao número e que o operador singular seleciona apenas entidades atômicas nessa denotação, podemos traçar um paralelo com o Karitiana. No entanto, como essa língua não conta com a diferenciação entre singular e plural nos nomes (cf. MÜLLER et al., 2006), tal operação seria realizada pelo morfema -ka do auxiliar tyka. A fórmula em (18a) e as ilustrações em (18b) e (18c) representariam a operação desse auxiliar sobre um nominal da sentença:

$$
\begin{aligned}
& \text { a. Tyka }=\text { ?P ?x[P (x) ??Atômico (x)] } \\
& \text { b. }[[\mathrm{ka}(\mathrm{CN})]]=\{\mathrm{a}, \mathrm{b}, \mathrm{c}, \ldots\} \\
& \text { c. }[[\text { Tyka (õwã) }]]=\{\tilde{o} w a ̃ 1, \text { õwã2 } 2 \text { õwã } 3, \ldots\}
\end{aligned}
$$

Considerando que o uso de um numeral como sypomp (dois) determina a semântica do sintagma nominal como plural, mesmo que não haja flexão no nome, e considerando nossa hipótese de que -ka é um operador singular sobre um dos nominais, podemos prever que uma sentença com um verbo transitivo marcada pelo auxiliar imperfectivo será agramatical se os dois argumentos forem antecedidos por sypomp. O operador singular contido no auxiliar imperfectivo não encontraria um nominal passível de ser singularizado. Observando os dados abaixo, constatamos que nossa previsão está correta:

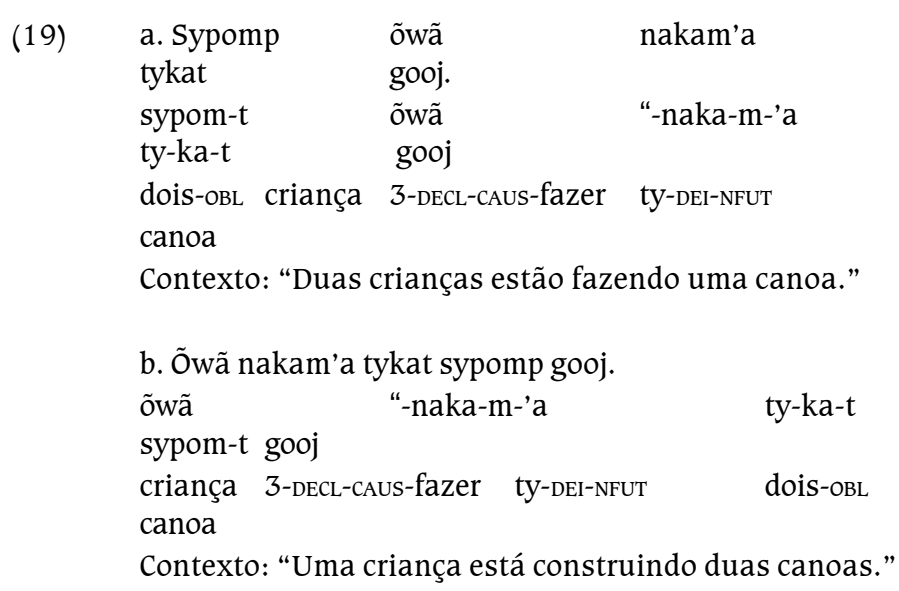




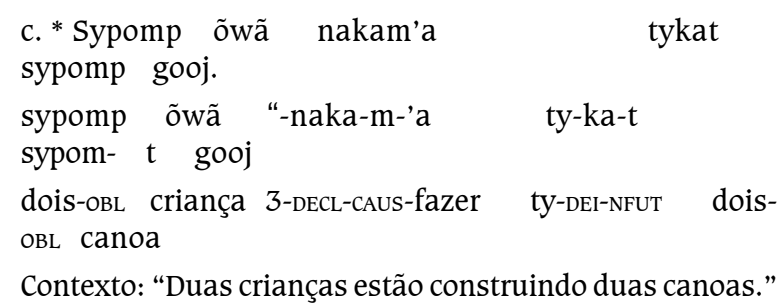

Observando o contraste entre as sentenças acima, podemos levantar a hipótese de que o tyka atua como um operador singular sobre um dos nominais, selecionando apenas entidades atômicas de sua denotação. Parece, portanto, que em Karitiana a distinção plural/singular dos NCs é determinada pelo núcleo aspectual. Podemos prever que, enquanto o tyka atua sobre os NCs como um operador singular, tysyp atua como um operador plural.

\section{A REPRESENTAÇÃo LóGICA DO TYKA}

O objetivo desta seção é apresentar uma forma lógica capaz de descrever toda a complexidade semântica do auxiliar tyka. Parsons (1989) traz uma proposta de representação formal para sentenças imperfectivas com base no inglês. Jóhannsdóttir (2006) adapta a forma lógica de Parsons (1989) para a descrição do islandês, língua em que o progressivo é construído com verbos posturais. Em sua fórmula, Jóhannsdóttir (2006) trata os eventos em andamento como estados. Nossa proposta é adaptar a forma lógica utilizada por Jóhannsdóttir (2006) para uma descrição do imperfectivo do Karitiana.

Adaptando as propostas de Parsons (1990) e de Jóhannsdóttir (2006), temos em (20) a representação lógica sugerida para uma sentença do Karitiana construída com tyka:

\section{(20) Õwã naka'y tykat kinda'o.}

$(? \mathrm{t})(\mathrm{t}=$ agora $\&(? \mathrm{~s})$ [em-movimento $(\mathrm{s}) \&$ comer $(\mathrm{s}) \&$ $\operatorname{ExArg}(\mathrm{s}$, criança) $\& \div \operatorname{ExArg} \%=1 \& \operatorname{InArg}(\mathrm{s}$, fruta) $\&$ Hold $(s, t)]]$

(Existe um tempo de referência que é simultâneo ao momento da fala e existe um estado que é "em-movimento" e que é de "comer". o argumento externo desse estado é criança, a cardinalidade desse argumento é igual a um, e o argumento interno é fruta. Esse estado descrito perdura no momento de referência, ou seja, o momento da situação contém o momento de referência) 
A representação lógica acima parece contemplar todas as nossas conclusões em torno da semântica do auxiliar tyka. Ela descreve não apenas a sua imperfectividade, mas também a sua possibilidade de indicação de eventos singulares ou plurais e a sua indicação da singularidade e da posição corporal da referência do nominal.

\section{CONCLUSÃo}

A proposta deste artigo era investigar a semântica do tyka do Karitiana. Nossa hipótese era de que seria um auxiliar imperfectivo compatível com eventos singulares e plurais, cujo morfema $-k a$ seria um operador singular sobre um SN. Além de confirmar essa hipótese, buscamos mostrar como o auxiliar em foco é incompatível com sentenças no passado e como pode ter seu morfema -ka substituído para indicar a postura de um SN.

Com base em nossa hipótese e nos conceitos oferecidos pela Semântica Formal, algumas previsões foram feitas. Inicialmente, de que uma sentença sem marcação aspectual deveria ter o mesmo número de SNs que uma sentença com tyka. Em seguida, de que sentenças com verbos estativos de conteúdo lexical não-temporário deveriam ser agramaticais com esse auxiliar. Foi previsto, também, que sentenças construídas com advérbios que implicam uma pluralidade de eventos deveriam ser gramaticais mesmo com o uso do tyka. Outra previsão era de que sentenças com esse auxiliar não poderiam ser gramaticais se contassem apenas com SNs semanticamente plurais. Finalmente, nossas previsões finais eram de que sentenças com advérbios indicadores de $\mathrm{TT}<\mathrm{TU}$ deveriam ser agramaticais com tyka, e que a troca de seu morfema -ka por -ja, -so e -syp representaria uma alteração na postura de um SN.

A análise relatada neste artigo mostrou que cada uma de nossas previsões estava correta, comprovando, assim, nossa hipótese.

\section{RESUMO}

Este trabalho investiga a semântica da palavra tyka do Karitiana. Nossa hipótese é de que se trata de um auxiliar aspectual compatível apenas com sentenças de TT $\geq$ TU e que representa a relação aspectual de TT $\underline{\mathrm{C}} \mathrm{TSit}$, correspondente na literatura ao imperfectivo (cf. KLEIN, 1994). Além de marcar a imperfectividade em sentenças no presente ou no futuro, tyka, mais especificamente seu morfema $-k a$, opera sobre um nominal, selecionando apenas as entidades atômicas de sua 
denotação. Além da complexidade semântica levantada em nossa hipótese, tyka pode expressar a posição corporal da referência de um nominal ao utilizar um outro morfema no lugar de -ka (cf. LANDIN, 1984).

Palavras-chave: semântica formal; aspecto verbal; Karitiana.

\begin{abstract}
This paper investigates the semantics of the word tyka in Karitiana. We defend that the word in focus is an aspectual auxiliary compatible only with sentences of TT $\geq$ TU and that it represents the aspectual relation of TT $\underline{\mathrm{C}} \mathrm{TSit}$, correspondent in the literature to the imperfective (Klein 1994). Besides marking imperfectivity in sentences in the present or in the future, tyka is an operator that acts over a nominal, selecting only atomic entities of its denotation. According to Landin (1984), it can also bring another morpheme instead of - $k a$ to express the body position of the reference of that nominal. Keywords: formal semantics; verbal aspect; Karitiana.
\end{abstract}

\title{
REFERÊNCIAS
}

CASTILHO, Ataliba T. de. Introdução ao estudo do aspecto verbal no português. Marília: Faculdade de Filosofia, Ciências e Letras, 1968. [Coleção Teses; separata de Alfa 12: 1967].

COMRIE, Bernard. Aspect: An introduction to the study of verbal aspect and related problems. Cambridge: Cambridge University Press, 1985.

DAVIDSON, Donald. Essays on actions and events. Oxford: Carendon Press, 1967.

EVERETT, Caleb. Gestural, perceptual, and conceptual patterns in Karitiana. Tese de Doutorado. Estados Unidos. Rice University, 2006.

HAEGEMANN, Liliane. Introduction to government and binding theory. 2. ed. Oxford, UK/ Cambridge, USA: Blackwell, 1995.

JÓHANNSDÓTTIR, K. Posture verbs in Icelandic. Disponível em: <http://docs.google.com/ viewer? $\mathrm{a}=\mathrm{v} \& \mathrm{q}=\mathrm{cache}: \mathrm{gMCPsQYLHhgJ}:$ ing.uwo.ca/publications/CLA2006/ Johannsdottir.pdf +http://ling.uwo.ca/publications/CLA2006/Johannsdottir.pdf\&hl=pt$\mathrm{BR} \& \mathrm{gl}=\mathrm{br \& sig}=\mathrm{AHIEtbRFqeg} \mathrm{FE}$ w9o-H309Ika4ipoaiLObw $>$

KLEIN, W. Time in language. London, New York: Routledge, 1994.

KRATZER, Angelika. On external arguments. Functional projections. GLSA, UMass, Amherst, 1994.

LANDIN, D. An outline of the syntatic structure of Karitiana sentences. Série Lingüística II, 1984. p. 219-254. 
MULLER, Ana; SANCHES-MENDES, L. Pluractionality in Karitiana._Sinn und Bedeutung, n. 12, Oslo, 2008. Proceedings of SuB 12, Oslo: Department of Literature, Area Studies and European Languages, University of Oslo, p. 442-454, 2008.

MÜLLER, Ana; STORTO, Luciana; COUTINHO-SILVA, Thiago. Number and the count-mass distinction in Karitiana. UBCWPL 19: Proceedings of the Eleventh Workshop on Structure and Constituency in Languages of the Americas, p. 122-135, 2006.

MÜLLER, A. Genericity and the denotation of common nouns in Brazilian Portuguese. In: WEERLE, Adam; KIM, Ji-Young (Eds.). The semantics of under-represented languages in the Americas. UMOP, n. 25, p. 72-80. Amherst, MA: GLSA, The University of Massachusetts, 2001.

PARAGUASSU-MARTINS, N.; MÜLLER, Ana. A distinção massivo-contável nas línguas naturais. Revista Letras, Curitiba: UFPR, 2008.

PARSONS, T. The progressive in English: events, states and process. Linguistics and Philosophy, n._12, p. 312-241, 1989.

Events in the semantics of English: A study in subatomic semantics. Cambridge, Mass.: MIT Press, 1990.

PORTNER, Paul H. What's meaning?: Fundamentals of formal semantics. Oxford: Blackwell Publishing, 2005.

REICHENBACH, Hans. Elements of symbolic logic. New York: Macmillan, 1947.

STORTO, Luciana R. Algumas categorias funcionais em Karitiana. In: CABRAL, A S. A. C.; RODRIGUES, A. D. (Orgs.). Línguas indigenas brasileiras. Fonologia, gramática e história. Tomo I. Belém: EDUFPA/UFPA, 2002. p. 151-164.

. Aspects of a Karitiana grammar. Boston, 1999. Tese (Doutorado) - MIT.

Enviado em: 27/10/2009

Aceito em: 27/01/2010 
